\title{
Large Area SEM Backscatter Imaging of GABA Post-Embedding Immunogold, Web-Based Crowdsourcing Segmentation and Local Image Processing for Rigorous Quantification
}

\author{
Cheryl Clarkson $^{1 *}$, Christopher T. Zugates ${ }^{2,}$ Christine A. Brantner ${ }^{1}$, Matthias Rust ${ }^{3}$ and Anastas \\ Popratiloff ${ }^{1}$ \\ 1. George Washington University, Nanofabrication and Imaging Center, Washington, DC, USA. \\ 2. arivis AG, Washington, DC, USA. \\ 3. arivis AG, Rostock, Germany. \\ * Corresponding author: cclarkson12@gwu.edu
}

Large-scale analysis of changes in the amount and localization of molecules in the brain using postembedding immunogold labeling was near to impossible due to spatial constraints of transmission electron microscopy (TEM) sample holders. In this study, we validate an approach enabling consistent production of very large area high-resolution scanning electron microscopy (SEM) images of target brainstem structures with localization and quantification of a specific neurotransmitter (Fig. 1A). The image quality and resolution supported hybrid segmentation, enabling quantification of the gold particles related to each manually drawn synaptic terminal (Fig. 1B and B'). The custom-designed analysis pipeline offered close to $100 \%$ accuracy in segmentation and quantification of gold particles. SEM multilayered image acquisition is a key element of this approach providing a solid framework to collect high-resolution image data based on lower resolution structural context image data relaying brainstem cytoarchitecture. We validated this approach using a logical pipeline based on commercial software (arivis AG) designed to segment and automatically quantify gold particles encoding GABA inside all labeled presynaptic terminals in the hypoglossal nucleus (Fig. 1C).

P7 old WT mice were anesthetized and then perfused with 4\% PFA and 0.5\% GA in 0.12M buffer. After post-fixation, $300 \mathrm{um}$ vibratome sections from the brainstems were cut and processed for EM embedding. Briefly, the brainstem sections were fixed in osmium tetroxide (1\%), infiltrated with uranyl acetate $(1 \%)$, dehydrated in ascending alcohols, cleared in propylene oxide and infiltrated with resin, followed by resin polymerization at $60^{\circ} \mathrm{C}$. After polymerization, sections containing the hypoglossal nuclei were trimmed and block-mounted. Ultrathin sections $(120 \mathrm{~nm})$ were cut and mounted on silicon wafer. To perform post-embedding immunogold labeling we used a conventional method for TEM [1]. Ultrathin sections were treated with $1 \%$ sodium metaperiodate and then incubated in rabbit anti-GABA and then a goat anti-rabbit IgG gold-conjugated, $10 \mathrm{~nm}$ diameter secondary antibody.

Immunogold-labeled brainstem sections were observed with a Helios NanoLab 660 SEM (Thermo Fisher, FEI) equipped with a concentric backscattering detector (CBS) or Through Lens Detector (TLD) using immersion mode. We compared different acquisition conditions, including $1 \mathrm{kV}, 2 \mathrm{kV}$, and $5 \mathrm{kV}$ voltage with landing current ranging from 100-800 pA. At $4.5 \mathrm{~mm}$ working distance the CBS detector provided the best signal differential between the uranium counterstain and the gold using $2 \mathrm{kV}$ and landing currents between 100-400pA. $100 \mathrm{pA}$ gave the best resolution of adjacent gold particles, but the signal of the uranium counterstain was weak at gain saturation of the detector. The major contributor to the signal was ring $\mathrm{B}$ of the CBS. Similar results were obtained with the TLD detector, but at a much shorter working distance. At $5 \mu$ s dwell time, CBS produced images with less white noise that the TLD backscattering mode. Therefore, the image acquisition was performed with CBS, at landing voltage of $2 \mathrm{kV}$ and current 
of $400 \mathrm{pA}$. High-resolution images $(5.18 \mu \mathrm{m}$ horizontal field of view, $1.6 \mathrm{~nm} / \mathrm{pixel}, 80 \mathrm{~K})$ were fused into a single tiling map per sample.

After converting the RAW format into a pyramidal redundancy-free format (Scalable Image Storage SIS) and transferring it to a server (arivis AG), all vesicle-containing profiles (boutons terminaux) were collaboratively manually segmented. They were then intersected with GABA-coding segmentation. The pipeline analysis for GABA immunogold labeling in SEM images comprised: 1) drawing all labeled presynaptic terminals. 2) application of a threshold, Gaussian blur, and a morphology filter. 3) masking synaptic boutons and inverse masking of mitochondria. 4) Isolation of the signal from gold particles via seed-based watershed segmentation. The final product is an object database containing the original image pixel locations. Morphometric parameters can be visualized at their original image location, while maintaining the relation of an object to overall population (Fig. 1C)

In the current study, we examined, the amount and distribution of GABA amino acid on the presynaptic terminals of the hypoglossal nucleus. A total of 5626 labeled presynaptic terminals were outlined in an area of 130x165 $\mu \mathrm{m}$, all them were segmented and analyzed using the pipeline, with a total of 204,886 gold particles quantified before Otsu thresholding [2]. After fixing the "background" value, a total of 1381 terminals were analyzed. The result was an interactive heat map showing the density distribution of GABA particles in the presynaptic terminals on the hypoglossal nucleus, excluding the mitochondria, allowing a fast and very accurate quantification of this neurotransmitter together with a spatial location of each segment traced using post-embedding immunogold labeling (Fig. 1C' and 1C').

References:

[1] C Clarkson, FM Antunes and ME Rubio, J. Neurosci. 36 (2016), p.10214.

[2] N Otsu, IEE Transaction on systems, Man, and cybernetics, SMC-9 (1979), p. 62.
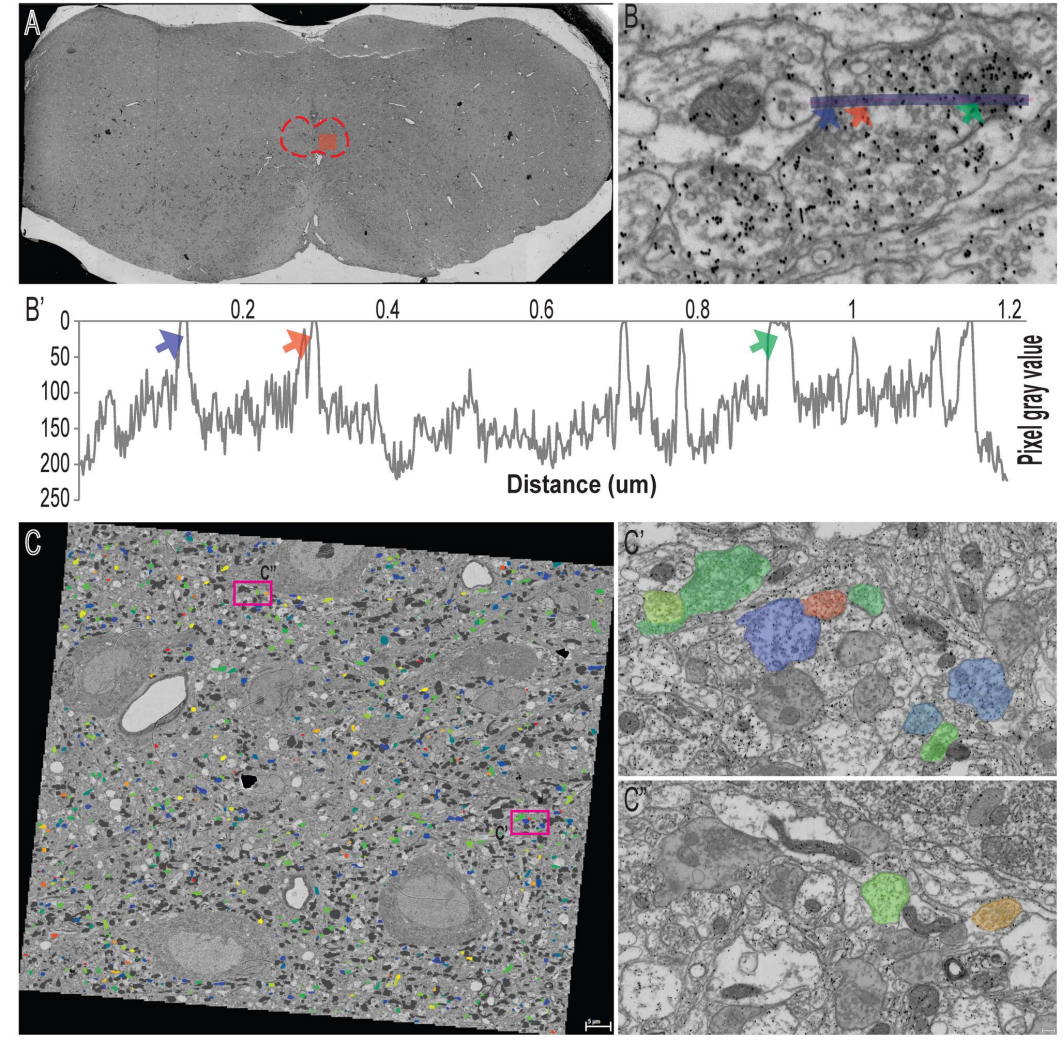

Figure 1. A) Hypoglossal nucleus location (red) in the mouse brainstem. B) Image showing a purple line in a labeled synaptic terminal, used to generate a pixel intensity scan (B'), arrows indicate the signal deriving from gold particles. Note the intensity differential between the uranyl counterstain and the gold particle. The morphology line scan plotted against pixel brightness illustrates that $1.6 \mathrm{~nm}$ pixel resolution is sufficient to resolve the signal registered from a single gold particle (blue arrow) by applying a single intensity threshold. This resolution is also supportive for intensity threshold separation of gold particles at close proximity to each other (red arrow) but not sufficient to resolve clusters (green arrow), justifying an additional application of morphology filter in addition to intensity threshold. C) Color coding-GABA terminal density distribution in WT mouse hypoglossal nucleus, C' and C' are high magnification $(80,000 \mathrm{x} / \mathrm{SFV})$ tile stitched SEM images of the XIIN highlighted in C, showing color coding distribution of all terminals where $10 \mathrm{~nm}$-gold particles were segmented, background level is represented in gray, low level in blue and green, medium level in yellow and orange and high density in red. 